\title{
Correction: PKCa is required for Akt-mTORC1 activation in non-small cell lung carcinoma (NSCLC) with EGFR mutation
}

This article has been corrected since Advance Online Publication and a correction is also printed in this issue

\author{
Mohamed F. Salama $\mathbb{1}^{1,2,3} \cdot$ Mengling Liu ${ }^{1,2,4} \cdot$ Christopher J. Clarke $^{1,2} \cdot$ Mel Pilar Espaillat ${ }^{1,2} \cdot$ John D. Haley ${ }^{5} \cdot$ \\ Ting $\operatorname{Jin}^{6} \cdot$ Daifeng Wang ${ }^{6,7} \cdot$ Lina M. Obeid (i) ${ }^{1,2} \cdot$ Yusuf A. Hannun $\mathbb{1}^{1,2,5}$
}

Published online: 1 October 2019

(C) The Author(s), under exclusive licence to Springer Nature Limited 2019

\section{Correction to: Oncogene}

https://doi.org/10.1038/s41388-019-0950-z

The original version of this Article was updated shortly after publication, because the Supplementary Information file was inadvertently omitted.
The original article can be found online at https://doi.org/10.1038/ s41388-019-0950-z.

Yusuf A. Hannun

yusuf.hannun@stonybrookmedicine.edu

1 Department of Medicine, Stony Brook University, Stony Brook, NY 11794, USA

2 Stony Brook Cancer Center, Stony Brook University Hospital, Stony Brook, NY 11794, USA

3 Department of Biochemistry, Faculty of Veterinary Medicine, Mansoura University, Mansoura, Egypt

4 Department of Immuno-Oncology, HD Biosciences Inc, San Diego, CA 92121, USA

5 Departments of Biochemistry and Pathology, Stony Brook University, Stony Brook, NY 11794, USA

6 Department of Biomedical Informatics, Stony Brook University, Stony Brook, NY 11794, USA

7 Department of Biostatistics and Medical Informatics and Waisman Center, University of Wisconsin-Madison, Madison, WI 53792, USA
The error has now been fixed and the Supplementary Information PDF is available to download from the HTML version of the Article. 